\title{
Local Recurrence of Retroperitoneal Paraganglioma Treated Laparoscopically
}

\author{
Ryuta Watanabe, MD, Akira Ozawa, MD, Tokuhiro Iseda, MD \\ Department of Urology, Matsuyama Shimin Hospital, Ehime, Japan (all authors).
}

\begin{abstract}
Introduction: Paraganglioma of the kidney seldom occurs and is rarely malignant. Because of anatomical characteristics and concerns about catecholamine release, surgery for these tumors is often deemed inappropriate. We present the case of a man with recurrent paraganglioma treated with laparoscopic resection.

Description: A 73-year-old man was referred to our department after an internal medicine check-up raised suspicion of a retroperitoneal tumor. Enhanced imaging revealed a $4.5-\mathrm{cm}$ tumor that surrounded the left hilus of the kidney. We performed a retroperitoneal laparoscopic resection of all of the tumor, and pathology confirmed a paraganglioma. Approximately 2 years after surgery, a CT scan showed an enlargement of the rest of the tumor around the left hilus of the kidney. We performed a retroperitoneal laparoscopic resection of the recurring tumor. With some difficulty we resected much of the tumor. There was no sign of recurrence 11 months after the second surgery, but long-term follow-up is essential.
\end{abstract}

Conclusion: Our case study is among only a few in which laparoscopic resection of a retroperitoneal paraganglioma has been reported. The findings collectively suggest that we should be careful of strong adhesion and sudden hypertension during such operations.

Key Words: Idiopathic kidney injury, Retroperitoneal laparoscopic resection, Retroperitoneal paraganglioma, Second operation.

\footnotetext{
Citation Watanabe R, Ozawa A, Iseda T. Local recurrence of retroperitoneal paraganglioma treated laparoscopically. CRSLS e2016.00105. DOI: 10.4293/CRSLS.2016.00105.

Copyright (C) 2017 by SLS, Society of Laparoendoscopic Surgeons. This is an open-access article distributed under the terms of the Creative Commons Attribution-Noncommercial-ShareAlike 3.0 Unported license, which permits unrestricted noncommercial use, distribution, and reproduction in any medium, provided the original author and source are credited.

Disclosures: None.

The authors thank the Ehime Urological Association for making publication of this case report possible through their financial support.

Address correspondence to: Ryuta Watanabe, Department of Urology, Matsuyama Shimin Hospital, Outemachi 2-6-5, Matsuyama City, Ehime 790-0067, Japan. Telephone: +91-89-943-1511, Fax +81-89-947-0026, E-mail: ryuta_w_pooh@yhahoo.co.jp
}

\section{INTRODUCTION}

Although laparoscopic surgery for pheochromocytoma has become popular, it has not gained wide acceptance for retroperitoneal paraganglioma because of the tumor's low incidence, its low degree of malignancy, challenging anatomic characteristics, and concerns over catecholamine release. Herein, we report a case of local recurrence of a retroperitoneal paraganglioma that we treated by performing retroperitoneal laparoscopic resection and provide discussion within the context of the existing literature.

\section{CASE REPORT}

A 73-year-old man was referred to our department after an internal medicine check-up raised the suspicion of a ret- roperitoneal tumor based on a plain computed tomography (CT) scan. Enhanced CT (Figure 1A) and magnetic resonance imaging (MRI; Figure 1B) revealed a tumor that was $4.5 \mathrm{~cm}$ in diameter surrounding the left hilus of the kidney, which did not connect to the renal adrenal gland. The metaiodobenzylguanidine (MIBG) scintigraphy did not show abnormal findings except for physiological accumulation lesion present (Figure 1C). Endocrinologic examination demonstrated slightly high levels of serum noradrenalin, serum aldosterone, urine noradrenalin, and urine dopamine. Specifically, the serum adrenaline level was $16 \mathrm{pg} / \mathrm{mL}$ (reference range, $0-100$ ), serum noradrenalin level was $576 \mathrm{pg} / \mathrm{mL}$ (range, 100-450), serum dopamine level was $9 \mathrm{pg} / \mathrm{mL}$ (range, $0-20$ ), serum cortisol level was $13.1 \mathrm{pg} / \mathrm{mL}$ (range, 6.24-18.0), serum 
Local Recurrence of Retroperitoneal Paraganglioma Treated Laparoscopically, Watanabe R et al.
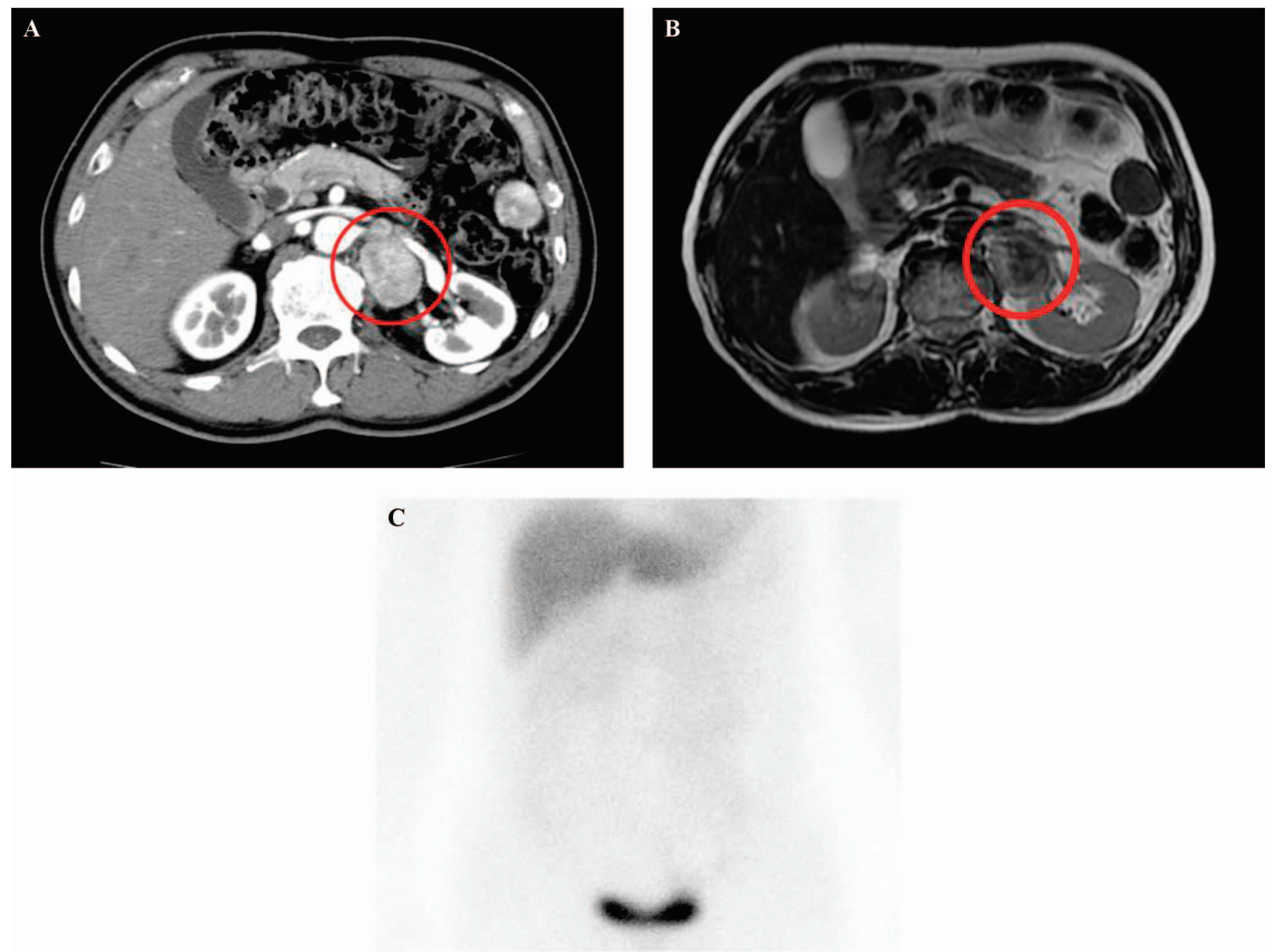

Figure 1. Enhanced CT A, and MRI B, revealed a tumor that was $4.5 \mathrm{~cm}$ in diameter around the left hilus of the kidney that did not connect to the renal adrenal gland. MIBG scintigraphy $\mathbf{C}$, did not show abnormal findings except for the physiological accumulation of the lesion.

aldosterone level was $576 \mathrm{pg} / \mathrm{mL}$ (range, 35.7-240) and serum adrenocorticotropic hormone (ACTH) level was $51.6 \mathrm{pg} / \mathrm{mL}$ (range, 7.2-63.3). In addition, the urine adrenaline level was $4.7 \mu \mathrm{g} /$ day (range, 3.4-26.9), urine noradrenalin level was $238.8 \mu \mathrm{g} /$ day (range, 48.6-168.4), urine dopamine level was $1641.3 \mu \mathrm{g} /$ day (range, 365961.5), urine vanillylmandelic acid (VMA) level was 2.4 $\mathrm{mg} /$ day (range, $1.5-4.3 \mathrm{mg} /$ day), urine metanephrine level was $0.05 \mathrm{mg} /$ day (range, $0.04-0.19$ ), and urine normetanephrine level was $0.19 \mathrm{mg} /$ day (range, $0.09-$ 0.33). The patient did not have any clinical symptoms, such as hypertension or hyperglycemia. Although MIBG scintigraphy was negative, we diagnosed this patient with retroperitoneal paraganglioma based on other data. Consequently, we performed a retroperitoneal laparoscopic resection of the tumor. Although the adhesion around the peripheral tissue was relatively strong, we were able to resect the entire tumor (Figure 2). There were no substantial changes in blood pressure during the operation. The operation took 3 hours and 14 minutes, and blood loss was $25 \mathrm{~mL}$. Histopathologic examination revealed the presence of paraganglioma. Immunohistochemical staining revealed Grimelius $(+)$, synaptophysin $(++)$, chromogranin, neuron-specific enolase (NSE), Leu-7, and S100 (focal + ), which are consistent with the characteristics of paraganglioma. The patient was discharged on the admission day 7 without difficulty. Subsequently, the patient was fol- 


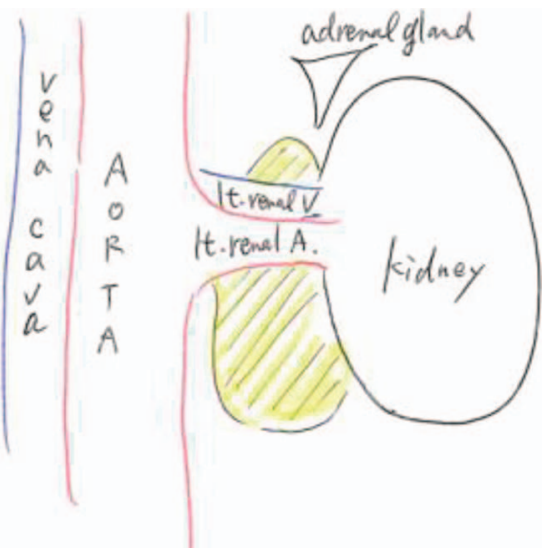

Figure 2. We performed a retroperitoneal laparoscopic resection of the tumor. Although the adhesion around the peripheral tissue was relatively strong, we were able to resect the entire visible tumor.

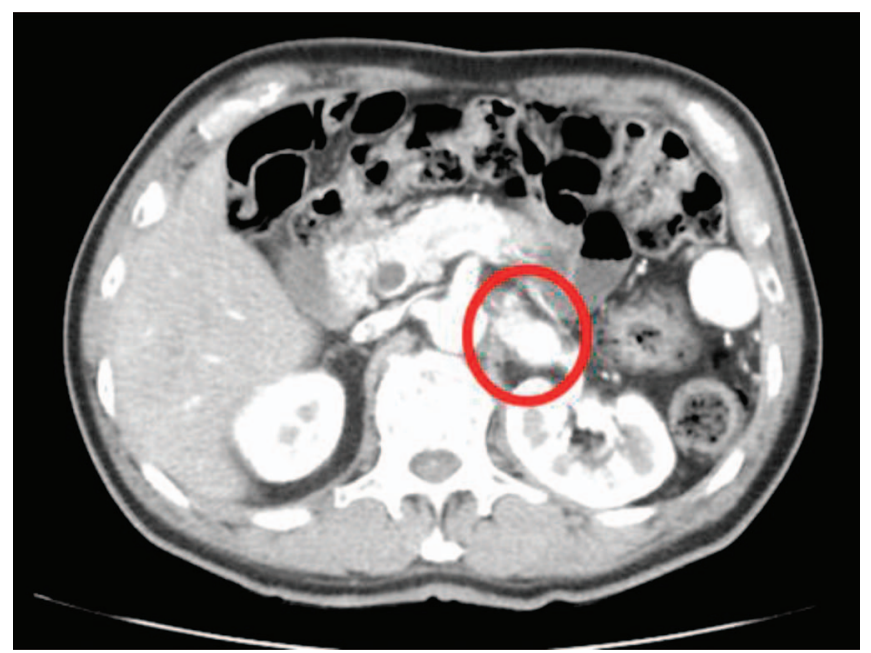

Figure 3. Approximately 2 years after surgery, CT demonstrated an enlargement of the tumor around the left hilus of the kidney.

lowed in the outpatient department. Approximately 2 years after surgery, CT demonstrated an enlargement of the tumor around the left hilus of the kidney (Figure 3), which we diagnosed as a tumor recurrence. The serum NSE level rose to $19.8 \mathrm{ng} / \mathrm{mL}$ (range, 0-16.3). Because the patient strongly preferred the resection of the rest of the tumor, we performed a second retroperitoneal laparoscopic resection of the recurring tumor. As we expected, the adhesion around the peripheral tissue was much stronger than that observed during the first operation, leading to an accidental cut into the left kidney. After the extension of the retroperitoneal cavity by an extension balloon was performed, the fiberscope was inserted into the space in front of the kidney because of

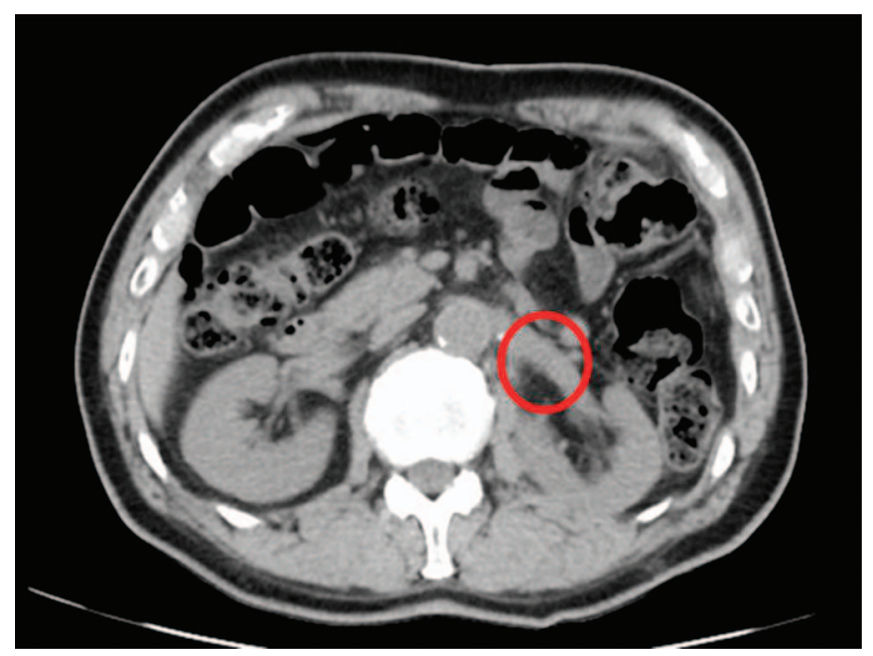

Figure 4. CT revealed no signs of recurrence 11 months after the second surgery.

the strong adhesion around the kidney, causing us to completely lose our orientation. We stopped the bleeding by soft coagulation with fibrin sealant patch and fibrin glue. We raised the kidney by snake retractor and sought for hilum of the left kidney. Then we identified the tumor in the upper side of the renal artery and we were able to resect the whole recurring tumor. The second operation lasted 5 hours and 49 minutes, with $950 \mathrm{~mL}$ of blood loss. Histopathologic examination demonstrated the recurrence of paraganglioma. Immunohistochemical staining revealed synaptophysin $(+)$, chromogranin A (+), NSE (+), CD57 (focal +), glial fibrillary protein (GFAP) (-), S100 (-) and MIB-1: 10\% of which are consistent with the characteristics of paraganglioma. The patient was discharged without any trouble on the 10th admission day. There were no signs of recurrence 11 months after the second surgery (Figure 4), but long-term follow-up is essential in the future.

\section{DISCUSSION}

Paraganglioma is a rare, benign neuroendocrine tumor that can arise from the adrenal medulla or the extraadrenal paraganglia. It is characterized histologically by chief cells with an abundance of neurosecretory granules that are arranged in lobules surrounded by sustentacular cells. Paragangliomas can arise anywhere in the sympathetic and parasympathetic chain of the ganglia with the most common site in the sympathetic nervous system being para-aortic. ${ }^{1}$ A paraganglioma may be of 2 types: (1) functional (sympathetic chromaffin paraganglioma/pheochromocytoma), as catecholamine-secret- 
ing tumors, hence appearing with typical symptoms such as paroxysmal hypertension, heart palpitations, headache attacks, and sweating or (2) parasympathetic nonfunctional neoplasias characterized as those lacking in chromaffin cells.

Paraganglioma is more common in men than in women and in individuals between 30 and 45 years of age. ${ }^{2,3}$ It can be detected early if clinical findings caused by excess secretion of catecholamine are present. The literature states that extra-adrenal retroperitoneal paraganglioma is functional in only up to $60 \%$ of patients. However, the data from other studies indicate that symptomatic hypertension is, in fact, more common. For those patients with nonfunctional extra-adrenal retroperitoneal paraganglioma, diagnosis usually depends on nonspecific factors related to the growth of a retroperitoneal mass. ${ }^{1}$ In the present case, endocrinologic examination demonstrated slightly high levels of serum noradrenalin, serum aldosterone, urine noradrenalin, and urine dopamine; however, the patient did not have symptoms that were caused by excess secretion of catecholamines. It is possible that in our case, we detected the growth of a retroperitoneal mass before the characteristic symptoms appeared.

Open resection is the gold standard for the surgical management of paraganglioma. The laparoscopic resection of paraganglioma is considered challenging for several reasons: the altered anatomic location, dense peritumoral adhesions, a high vascularity envelope, and proximity to major blood vessels. Because of the low incidence of the tumor, only a few studies of laparoscopic surgery for paraganglioma have been reported, most of which were single-case reports or limited case series studies. To the best of our knowledge, most of these studies reported successful removal of the paraganglioma but simultaneously conveyed longer operative times and a higher incidence of complications. Compared with the transperitoneal laparoscopic approach, which is commonly used to resect retroperitoneal tumors, the retroperitoneal laparoscopic approach has a few advantages, including shorter operative times, fewer disturbances of abdominal organs, and more rapid convalescence. ${ }^{4}$ In the present case, just for the first operation, the retroperitoneal laparoscopic approach was a good approach as noted by several factors, including less blood loss, relatively short operative time, and good visibility of tumors. However, during the second operation, we encountered difficulties in safely resecting the tumor because of strong peritumoral adhesions. After the extension of the retroperitoneal cavity by an extension balloon was performed, the fiberscope was inserted into the space in front of the kidney, and then we should have peeled off the peripheral kidney from the front side to the back side of the kidney. However, at that time, we unintentionally cut deep into the kidney tissue. We considered the possibility of the en bloc resection with sacrifice of the kidney before the second surgery, and obtained the consent of the patient and the family beforehand. Fortunately we were able to preserve the left kidney, although it incurred some injury. If further life-threatening complications had occurred, we may have had to perform a nephrectomy. We may have had better success with an open and intraperitoneal approach. In our opinion, retrospectively, it was unreasonable to use a laparoscopic approach in the second operation, but the patient's strong preference was to undergo a laparoscopic operation rather than open surgery. Even if we had selected an intraperitoneal laparoscopic approach, we thought the situation would be the same. Because the tumor was located behind the renal hilus, we could have hardly to reach the tumor because of the strong tissue adhesion.

It would be absolutely impossible for the patient to undergo a third operation. If the patient has another tumor recurrence, we will recommend hand-assisted techniques or open surgery. Apart from surgery, treatment options will be MIBG radiotherapy or CVD chemotherapy (cyclophosphamide, $750 \mathrm{mg} / \mathrm{m}^{2}$ body surface on day 1 ; vincristine, $1.4 \mathrm{mg} / \mathrm{m}^{2}$ body surface on day 1; and dacarbazine $600 \mathrm{mg} / \mathrm{m}^{2}$ body surface on days 1 and 2, repeated every 21 days).5 MIBG radiotherapy did not have an effect in the present case because the MIBG scintigraphy was negative.

In the initial operation, we thought that we could resect the whole tumor completely under a good field of view by a retroperitoneal approach. However, we should follow up this case carefully, because paraganglioma, particularly malignant paraganglioma, sometimes recurs in the same or other areas or metastasizes to other organs. There were still no signs of recurrence 11 months after the second surgery, but long-term follow-up by image diagnosis and the tumor marker NSE is essential for effective follow-up.

\section{CONCLUSION}

We report a case of local recurrence of retroperitoneal paraganglioma. We were able to successfully resect the tumor initially by laparoscopy, but our findings suggest that reoperation of a recurring tumor is unreasonable and inadvisable. Based on our experience, we recommend an 
open operation. Furthermore, long-term follow-up by image diagnosis and the tumor marker NSE are essential.

\section{References:}

1. Haye WS, Davidson AJ, Grimley PM, Hartman DS. Extraadrenal retroperitoneal paraganglioma: clinical, pathologic, and CT findings. AJR Am J Roentgenol. 1990;155:1247-1250.

2. Mikhail RA, Moore JB, Reed DN Jr, Abbott AR. Malignant retroperitoneal paragangliomas. J Surg Oncol. 1986;32: $32-36$.
3. Enzinger FM, Weiss SW. Paraganglioma. In: Enzinger FM, Weiss SW, eds. Soft Tissue Tumors. St. Louis: Mosby, 1988: 836-860.

4. Weifeng Xu, Hanzhong Li, Zhigang Ji, et al. Retroperitoneal laparoscopic management of paraganglioma: a single institute experience. PloS One. 2016;11:e14943.

5. Fliedner SMJ, Lehnert H, Pacak K. Metastatic paraganglioma. Semin Oncol. 2010;37:627-637. 